Proceedings

\title{
Infoculture Methods ${ }^{\dagger}$
}

\section{Bo Gao}

Software Engineering Institute, East China Normal University, Shanghai 200062, China; gaobo@sei.ecnu.edu.cn

† The Fourth International Conference on Philosophy of Information, Berkeley, CA, USA, 2-6 June 2019.

Published: 13 June 2020

\begin{abstract}
In big data, we know that it is important to have enough data to analyze, but where can one get more data? How does one get useful information? My novel idea of "infoculture" uses new BIONICS to resolve the problem of how to produce information for the artificial intelligence (AI) industry. Data can be cultivated by humans, just like how humans cultivate fish or shrimp. In the field of infoculture, the network is like an ocean, and the information machine is the fish and shrimp. However, key problems within this field are DATA DNA engineering, information disease engineering, infoculture engineering, and the ecology of information. The Google self-driving car is an example of a cultivated fish in infoculture theory.
\end{abstract}

Keywords: infoculture; protein type of data; computing agriculture; information philosophy; information bionics

\section{Introduction}

In the past few decades, the information industry always has been considered part of computer science. This includes operating systems, databases, and software development subjects often used. Currently, due to the information industry upgrade to the artificial intelligence (AI) industry, I found this industry not only in need of digital hardware and software, but also in need of more important factors - what is enough information and useful data producing. How can we get more information? How can we get more useful information we need? Computing is just a mathematical methodology used to analyze information by a computer program using code. Mathematical methodology is a result-oriented information processing method, but it is impossible to predict the source of information and the intelligence of information like a human being could. Therefore, we like to use a perspective from the field of information philosophy, which also has the most concern regarding modern AI science. In this study, I was concerned with the most comprehensive way to examine the AI new industry and traditional information technology (IT) industry. Computing science is not enough to fix these industries, therefore we need a new epistemology: a comprehensive 'infoculture' theory could be this.

\section{The Traditional Conception of Information Academic Discipline}

According to WIKI definition, computer science [1] includes the following areas: computer science calculation theory, algorithms and data structures, programming languages, compilers, parallel and distributed systems, software engineering, system structure, communication and network, database system, artificial intelligence, computer graphics, human-computer interaction, and scientific computing. Many modern businesses have benefited from the conception of IT industry, e.g., Java, Oracle, Microsoft, Cisco, Huawei, etc., and in turn, their technology practices have grown into different theories of computer science. Because the IT industry grows very fast, many technologies are continually being improved and updated after their creation. For example, operating 
systems (OS) are a basic program system used to operate the hardware. An OS gives lifeless hardware the ability to think, in the same way, philosophy can inspire human thought. Networks are basic infrastructure, and TCP/IP is the language used to run the whole internet. IT is based on binary data system - one mathematics language - and like in society, language helps every computing hardware device with an OS communicate with one another. Many applications that are developed used coding can be run as an automated program, and generate all kinds of data to meet the needs of the human being programming it. The IT industry is a crucial part of everyday life-it can take on many repetitive tasks, and help us save time and avoid risk. But one questions remains: Does computer science provide a suitable and comprehensive theory of the IT industry and AI industries?

I argue that it does not. IT hardware, such as computer devices, smartphones, switches, routers, printers, cables, all belong within the domain of electronics manufactures. Within this domain, there is no need for mathematical language, so why we apply computer science theories to this field? This is a significant issue. Network activity is the practice of computing language communicating between different network devices via TCP/IP, which is built on OSI7 layer model. However, the network infrastructure is also a hardware system from an electronics manufacturer. Therefore, computer science theories may not be totally applicable. Database management is a very important part of the IT industry. The data in the database is structured data, and a database is a structured storage system. This means they can store information produced by different applications within the hardware of a computing device. It is important to consider a database as an information system, rather than a computer system, but this also means a computing theory may not be applicable in this case either.

Computer science theories are not applicable to all aspects of the IT industry, and applying an incomputable theory can create problems. In university, it is difficult to define computer science, and many institutes often offer two different degrees in order to separate the different aspects of computer science. These are an "academic degree" and a "professional degree" [2]. Academic degree focuses on course credits, scientific research quality credits, and developing the skills of scientific and technological innovation. Whereas, professional degree use a curriculum system that focuses on course credits, professional ability credits, and practice credits.

\section{Professional Practice in Modern IT Industry}

Professional practice is always focused on a popular technical skill of the IT industry, often concerning the IT company`s product. However, many companies do not create computer functions any more, such as MCSE [3], ERP [4], ITIL [5], TOGAF [6], CISA [7], and ISO27001 [8].

MCSE is a technic knowledge system invented by Microsoft that manages all windows in an OS; it is very popular on the most client system and data servers. ERP is a corporate organization digital thinking methodology, which also supports ERP technic software system; nearly all 500 Fortum enterprises use it. ITIL is a type of framework used for IT service knowledge; it was invented in the $\mathrm{UK}$, and is very popular in the global IT industry; also, nearly all of 500 Fortum enterprises use it as a basic management thinking method, often called IT MBA. TOGAF is an advanced architecture method for organization enterprises; it uses a management philosophy that is completely different from computer science theories. CISA and ISO27001 are both popular international methods used to secure information management systems for all business organization; it is completely different from computer science theories, but constantly used by most of the IT industry.

These all conceptions are necessary and important, but belong to the field of management science, not computer science. This is different from traditional management theory, which advocates for an interdisciplinary approach to digital thinking based on IT technology. However, if we keep computer science philosophy limited to computing science theory, how can we apply our findings to the modern IT industry, and emerging AI industry?

\section{The Conception of Information Academic Discipline: AI}

AI was first proposed in 1956, but AI is a big broad term, and there is no clear agreement on its definition so far. Ref [9] At its simplest, AI does not rule out the ability to replace people with computing techniques, or the integration of others. It is difficult to define AI science, but I can confirm 
that the modern IT industry includes an emerging AI industry. The AI industry includes: deep learning, convolutional nerve retention, self-coding, embedding, artificial neural networks, supervised learning, feature learning, regularization, generation of antagonistic networks, generation of models, transfer learning, reinforcement, anomaly detection, inference, annotation, etc. [10]. One of the main differences between the IT and AI industry is regarding data research, which is often a main focus of the AI industry. Unlike the IT industry, hardware and software are not the most important factors in this field, even though the data needs hardware and software. IT devices produce data, and AI produces information-and the data only becomes valuable to us after it has been processed.

\section{The Novel Theory of Infoculture as Applied in the AI Industry}

From studying both the IT industry and AI industry, I found computer science theories are too confusing. They are too widely applied, and are not robust enough to support the epistemology of the information industry. However, I propose 'infoculture' to breach this gap.

In the age of AI, the smart machine needs in the same language as the software, which keeps accepting external data and produces new data by rational LOGOS [11]. The LOGOS is called the "Architecture Model" in the IT industry, and can be considered as DNA to an AI robot. The new useful DATA is like "nutrition" for this robot. Therefore, I have called this "Protein Type of Data" (PTD) (because like protein is crucial in the diet of a human, PTD is crucial in the diet of AI. From the DIKW model(Data/Information/Knowledge/Wisdom), we know that information is useful data, so information is PTD here. With enough information, and AI's rational thinking can produce knowledge, much like humans can produce wisdom through philosophical thought.

In big data, it is important to have enough data to analyze, but where can one get more data? How does one acquire useful information? Infoculture uses new information BIONICS to resolve this problem: Data can be farmed by humans, much like humans farm other resources (such as shrimp).

Infoculture regards a network like an ocean-and the information machine is the shrimp. However, key issues within this ocean are DATA DNA engineering, information disease engineering, infoculture engineering, and the information ecology. For example, Google's self-driving car can be considered a cultivated fish within an infoculture epistemology. The 'shrimp' that Google produces contains a lot of useful information after millions of automatic-driving miles . This is called testing data, or PTD.

Humans come from the ocean, and humans will return to the ocean, but these oceans are different-one is an ocean of water, the other an ocean of data.

\section{Conclusions}

In summary, the theories and epistemology of computer science cannot be globally applied to all areas of the IT and AI industries. My novel concept of 'infoculture' can breach the gap and be applied to every field. Infoculture a data cultivation bionics conception for the AI industry, and PTD is one of it's key ideas.

Conflicts of Interest: The authors declare no conflict of interest.

\section{References}

1. Computing Science. Available online: https://en.wikipedia.org/wiki/Computer_science (accessed on 3 February 2019).

2. Chai, Z.; Li, Y. Exploration and Practice of Classified Cultivation of Masters in Computer Science Oriented by "Harmony in Diversity". Sci. Educ. Artic. Collect. (XIA XUN) 2017, 12, 55-56.

3. MCSE. Available online: https://en.wikipedia.org/wiki/mcse (accessed on 1 February 2019).

4. ERP. Available online: https://en.wikipedia.org/wiki/erp (accessed on 1 February 2019).

5. ITIL. Available online: https://en.wikipedia.org/wiki/itil (accessed on 1 February 2019).

6. TOGAF. Available online: https://en.wikipedia.org/wiki/togaf (accessed on 1 February 2019).

7. CISA. Available online: https://en.wikipedia.org/wiki/cisa (accessed on 1 February 2019). 
8. ISO27001. Available online: https:/en.wikipedia.org/wiki/iso27001 (accessed on 1 February 2019).

9. AI. Available online: https://en.wikipedia.org/wiki/Artificial_intelligence (accessed on 1 February 2019).

10. Li, H.; Chen, Z.; Zhu, W. Practice and Thinking of Decision-making Intelligence Analysis Service Under the Situation of "First-Class" Discipline Construction-Taking the Gap Analysis of Artificial Intelligence Research Between China and the United States for Instance. J. Intell. 2019, 1, 1-7.

11. Wang, Z.S.; Fan, M.S. History of Greek Philosophy; People's Publishing House: Beijing, China, 1997; Volume 1, p. 445.

(C) 2020 by the authors. Licensee MDPI, Basel, Switzerland. This article is an open access article distributed under the terms and conditions of the Creative Commons Attribution (CC BY) license (http://creativecommons.org/licenses/by/4.0/). 